\title{
Artifacts in Neuroimaging - Pitfalls in Volume Electron Microscopy for CLEM and in Freeze-Fracture Replica Immunogold Labeling -
}

\author{
Naomi Kamasawa ${ }^{1}$ \\ 1. Electron Microscopy Core Facility, Max Planck Florida Institute for Neuroscience, Jupiter, USA
}

Correlative light and electron microscopy (CLEM) has recently been re-acknowledged in Neuroscience as a powerful tool to study structure and function of the nervous system. How to correlate highresolution images taken with electron microscopes (EMs) with a functional live fluorescence imaging would be challenging especially in complex brain tissues. We established a new CLEM workflow between 2-photon microscope and EM to investigate ultrastructural changes of functionally modulated spines using GFP as a bridging molecule [1]. While for the analysis of membrane proteins such as channels and receptors, the freeze-fracture replica immunogold labeling method has huge advantage to visualize arrangements and distributions of the target proteins in a two dimensional manner, and by using membrane bound GFP, replica images can be correlated to functional fluorescence images as well. As we all know, there are many artifacts existing in all EM procedures. In this paper, I would like to share and discuss new types of artifacts that we have been facing since we introduced new technologies and workflows.

ATUM/SEM: To obtain volume EM images to correlate with 2-photon live images, we established a workflow using an automatic tape-collecting ultramicrotome, ATUMtome (RMC/Boeckeler) and a scanning electron microscope (SEM) with an automatic array tomography software, Atlas 5AT (Carl Zeiss) [1]. A relatively large block face size of 1 x $1.5 \mathrm{~mm}$ that can cover a half of a hippocampus vibratome slice embedded in resin was cut, and the sections were collected on a roll of Kapton tape automatically. An anti-static tool for sectioning was helpful to serially cut sections and to align them on the tape. The surface of the Kapton tape was sometimes scratched during its production, and was also sometimes dirty with debris, which required pre-selection and cleaning of the tape. In addition, discharging of the tape surface was crucial to avoid wrinkles on the sections. A certain amount of compression of the ultrathin sections was evidence even by using a 35-degree knife, and chattering became more obvious when handling such larger samples. Before SEM observation, sections on the tape had to be electron conductive by carbon coating and/or carbon painting. These small tips were essential to serially image large enough area of interest from hundreds of sections with minimum artifact. Even when we carefully prepared the tools, equipment and samples, the sections on the tape were yet impossible to be perfectly flat, and manual "preview" of the imaging area before starting automatic $\mathrm{SEM} /$ Atlas image acquisition was useful to confirm focus and stigmatism in each imaging position.

Freeze-fracture replica immunogold labeling: These are many artifacts known in the freeze-fracture replica method [2]. With applying immunogold labeling on the top of the conventional replica protocol [3], new artifacts are potentially added and make the analysis harder. By using primary and secondary antibodies to label target proteins, a labeling uncertainty of ca. $30 \mathrm{~nm}$ from the epitope has to be always remember [4], and shapes of intramembrane proteins/particles (IMPs) seen as "bumps" in replica images become important to analyze the distributions of the marker immunogold particles. However, the shapes and distributions of IMPs can be easily altered by artifacts of the sample preparation; the temperature of both fracturing and material evaporations changes the quality of replicas, and water/ice contamination changes the appearances of IMPs. Coating thickness affects labeling efficiency as well. Unfortunately, 
sizes of commercial immunogold particles conjugated with secondary antibodies are sometimes not homogeneous enough to be used for double labeling procedure, where two different sizes have to be differentiated. Insufficient SDS-digestion also creates non-specific labeling, and careful evaluation of the replica quality is always important.

Summary: In addition to traditional artifacts in the entire EM sample preparation process, we have to face many more new artifacts when we handle new methodologies. All artifacts could be overcome, but a huge effort is always required for each artifact. We need to try to precisely understand what the artifacts are, and carefully pursue the image analysis.

\section{References:}

[1] N Kamasawa et al, Microscopy and Microanalysis 21 (2015), 1271-72.

[2] JE Rash, CS Hudson, "Freeze Fracture: Methods, Artifacts, and Interpretations" (Raven Press)

[3] K Fujimoto. Journal of Cell Science 108 (1995), 3443-3449.

[4] N Kamasawa et al, Neuroscience. 142 (2006), 1093-1117.
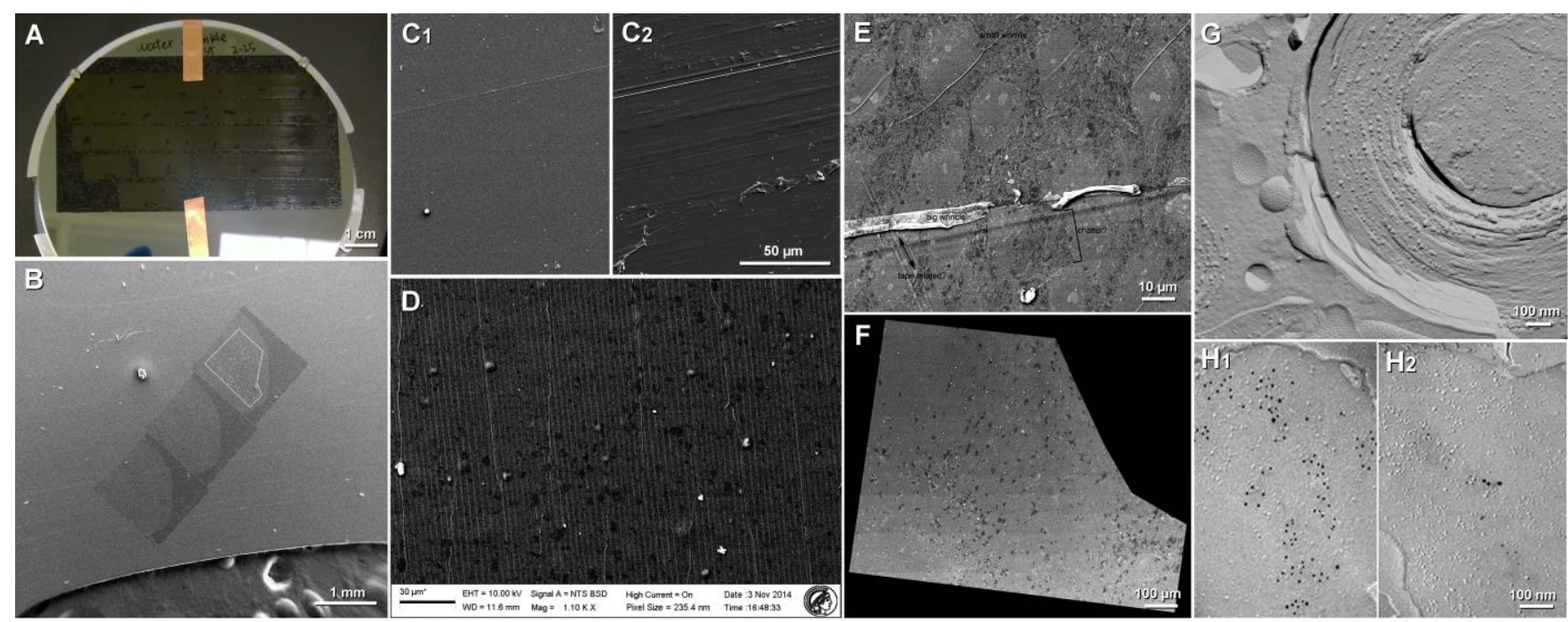

Figure 1. A) Silicon wafer prepared for the ATUMtome/SEM array tomography workflow. Pieces of Kapton tape having ultrathin sections are aligned on the wafer with carbon adhesive tape. Two pieces of copper tape were used for grounding the silicon wafer containing the sections to the SEM specimen holder. B) Low-magnification SEM image of three sections on Kapton tape. These sections were rotated when they were picked up on the tape. C) SEM images of a relatively smooth and clean surface (left) vs. a rough and dirty surface (right) of Kapton tapes. D-E) Chatters and wrinkles were seen on a section under SEM observation with low magnification (D) and high magnification (E) images. F) A montaged SEM image of the white-boxed area in the section in Fig. B. The image was captured with no tape damage, chatters or wrinkles, but small electrical charging was evident, which changed image brightness gradually among the tiling process. G) Freeze-fractured image of myelin. Lumpy organelle membranes and small crystals on the cross-fractured myelin layers are seen as a result water contamination. $\mathrm{H}$ ) An example of non-uniform size of immunogold particle (left) and a good double-labeled example with two sizes of immunogold particles (right). Note there are electron dense regions in the replicas indicating insufficient SDS-digestion. 